\title{
Dynamic of resistance alleles of two major insecticide targets in Anopheles gambiae (s.l.) populations from Benin, West Africa
}

\author{
Benoît S. Assogba1,2,3,4*, Nicole Pasteur', Patrick Makoundou' ', Sandra Unal', Lamine Baba-Moussa²,
} Pierrick Labbé ${ }^{1}$ and Mylène Weill ${ }^{1}$

\begin{abstract}
Background: Insecticide resistance is a growing concern for malaria control and vector control effectiveness relies on assessing it distribution and understanding its evolution.

Methods: We assessed resistance levels and the frequencies of two major target-site mutations, L1014F-VGSC and G119S-ace-1, conferring resistance to pyrethroids (PYRs) and carbamates/organophosphates (CXs/OPs) insecticides. These data were compared to those acquired between 2006 and 2010 to follow resistance evolutionary trends over ten years.

Results: We report the results of a 3-year survey (2013-2015) of insecticide resistance in 13 localities across the whole country of Benin. Permethrin (PYR) resistance was found in all populations tested, L1014F-VGSC being almost fixed everywhere, while bendiocarb resistance was limited to a few localities, G1 19S-ace-1 remaining rare, with very limited variations during surveyed period. Interestingly, we found no effect of the type of insecticide pressure on the dynamics of these mutations.
\end{abstract}

Conclusions: These results confirm both the high prevalence of PYR resistance and the potential of CXs/OPs as short- to medium-term alternatives in Benin. They also underline the need for regular resistance monitoring and informed management in their usage, as the G119S-ace-1 mutation is already present in Benin and surrounding countries. Their unwise usage would rapidly lead to its spread, which would jeopardize PYR-resistant Anopheles control.

Keywords: Anopheles gambiae, Malaria, Insecticide resistance, Evolution

\section{Background}

Controlling mosquito vectors of human diseases is a major health issue, particularly in sub-Saharan Africa where Anopheles gambiae (s.l.) is the main vector of malaria transmission. Malaria control is mainly based on insecticide use in the form of LLINs (long-lasting insecticide nets) and IRS (indoor residual spraying) [1].

\footnotetext{
${ }^{*}$ Correspondence: assobe80@gmail.com

${ }^{1}$ Institut des Sciences de I'Evolution de Montpellier (ISEM), UMR

CNRS-IRD-EPHE-Université de Montpellier, Place Eugène Bataillon, 34095 Montpellier, France

Full list of author information is available at the end of the article
}

Pyrethroid insecticides (PYRs) have been the cornerstone of malaria prevention in Africa for almost two decades, after Abuja Declaration in 2000, when Benin and the rest West African countries set-up a proper plan against malaria control [2]. However, the ability of vectors to survive insecticide treatments (i.e. become resistant) is a growing concern, as it may threaten vector control effectiveness for preventing malaria transmission [3-6]. Insecticide resistance is indeed widespread in natural populations in West Africa and more particularly in Benin, Burkina Faso and Ivory Coast [7-9].

In Benin, the Ministry of Health $(\mathrm{MOH})$ and the National Malaria Control Programme (NMCP) started 
a large and extensive distribution programme of pyrethroid-treated LLINs across the country in 2008 [2]. Moreover, through the USA PMI programme (President Malaria Initiative), large-scale indoor spraying campaigns are implemented since 2008, using carbamate (CXs) and organophosphate (OPs) insecticides [10].

In addition to this insecticide pressure due to malaria vector control, insecticides are widely used in agriculture for crop protection. Agricultural pesticides often belong to the same classes as those used for vector control $[7,11]$. Thus, they can affect the susceptibility of vector populations by increasing the frequencies of existing resistance mechanisms, or by selecting new mechanisms [11-14]. Because these selective pressures are heterogeneous, resistance frequency and its effect on vector control could vary from one population to another and over time [12].

This situation called the World Health Organization (WHO) to publish a Global Plan for Insecticide Resistance Management (GPIRM) [15] in 2012 (recently extended by guidelines at the national level), which emphasized the need for improved strategies for insecticide resistance management and prevention [16]. This objective cannot be achieved if the forces driving insecticide resistance evolution are not clearly understood and monitored.

Insecticide resistance dynamics, and thus long-term efficacy of vector control strategies, indeed depends on the biological characteristics of vectors, and the intensity and spatio-temporal distribution of selection pressures. It is therefore crucial to monitor the general patterns of resistance allele variations in relation with the various selective pressures that could drive these variations in vector populations. In the present study, we performed an updated overview of resistance status of An. gambiae (s.l.) in different agroclimatic zones with varying practices of insecticide across the whole Benin country. We acquired relevant information regarding selection pressure sources and its evolution to assess how they could contribute to the selection of two major target sites resistance mechanisms (the L1014F-VGSC and G119S-ace-1 mutations, respectively for PYRs and CXs/OPs resistance). These two mutations are the major one involved into PYR and CXs/OPs resistant in An. gambiae (s.l.) in West Africa. However, other SNPs have been recently identified in the voltage gate sodium channel gene (VGSC), but data on their role in insecticide resistance are lacking $[17,18]$. We provide encouraging data for CXs and OPs uses as means to circumvent the high An. gambiae (s.l.) PYR resistance in Benin for short- and medium-term.

\section{Methods}

Study sites and mosquito collections

This study was carried out in 13 localities following a longitudinal transect across Benin (Fig. 1): five localities in the southern part, characterized by a Guinean bioclimate (two rainy seasons, April-July and September-November and an average annual rainfall of $1500 \mathrm{~mm}$ ); four localities in the central part, characterized by a SudanoGuinean bioclimate (two rainy seasons, April-July and September-November with an average annual rainfall of $1000 \mathrm{~mm}$ ); and four localities in the northern part, characterized by a Sudanese bioclimate (one rainy season, June to October and an average annual rainfall of 900 $\mathrm{mm})$. These localities were selected according to insecticide use for crops protection or for malaria vector control and priority was given to localities involved in previous studies, to assess resistance dynamics (Table 1): (i) seven localities (Cotonou, Grand-Popo, Bohicon, Abomey, Glazoué, Bembèrèkè and Kandi), where vegetables and cotton are produced, with strong economic interests for the farmers and government, are thus mainly exposed to agricultural treatments; (ii) four localities (Tori-Bossito, Avrankou, Djougou and Natitingou) are less exposed to these agricultural insecticides, but have been selected for specific experimental malaria vector control intervention (MVCI), with more intense vector control interventions, i.e. more frequent turnover of bednets and IRS implementation; and (iii) two localities have little insecticide exposure, with limited insecticides usage for domestic production or public-health (Covè and Comè).

Anopheles gambiae (s.l.) larvae were sampled in these localities over three years (2013-2015). Note that due to field conditions and availability of the larvae during the sampling campaign, we were not able to collect a sample in each locality each year. At least 2000 larvae were collected from different breeding sites within a 1-km radius, in order to have enough adults per locality for the different tests included into this study.

\section{Species identification}

All emerged adult mosquitoes were assigned to the $A n$. gambiae (s.l.) cryptic species complex using morphological and molecular tests [19-21] after DNA extraction from whole mosquito as described in Assogba et al. [22].

\section{Insecticide susceptibility bioassays of An. gambiae (s.l.) populations}

Susceptibility assays were implemented according to the WHO protocol using 2- to 5-day-old mosquitoes [23]. When enough adults were available, four replicates 


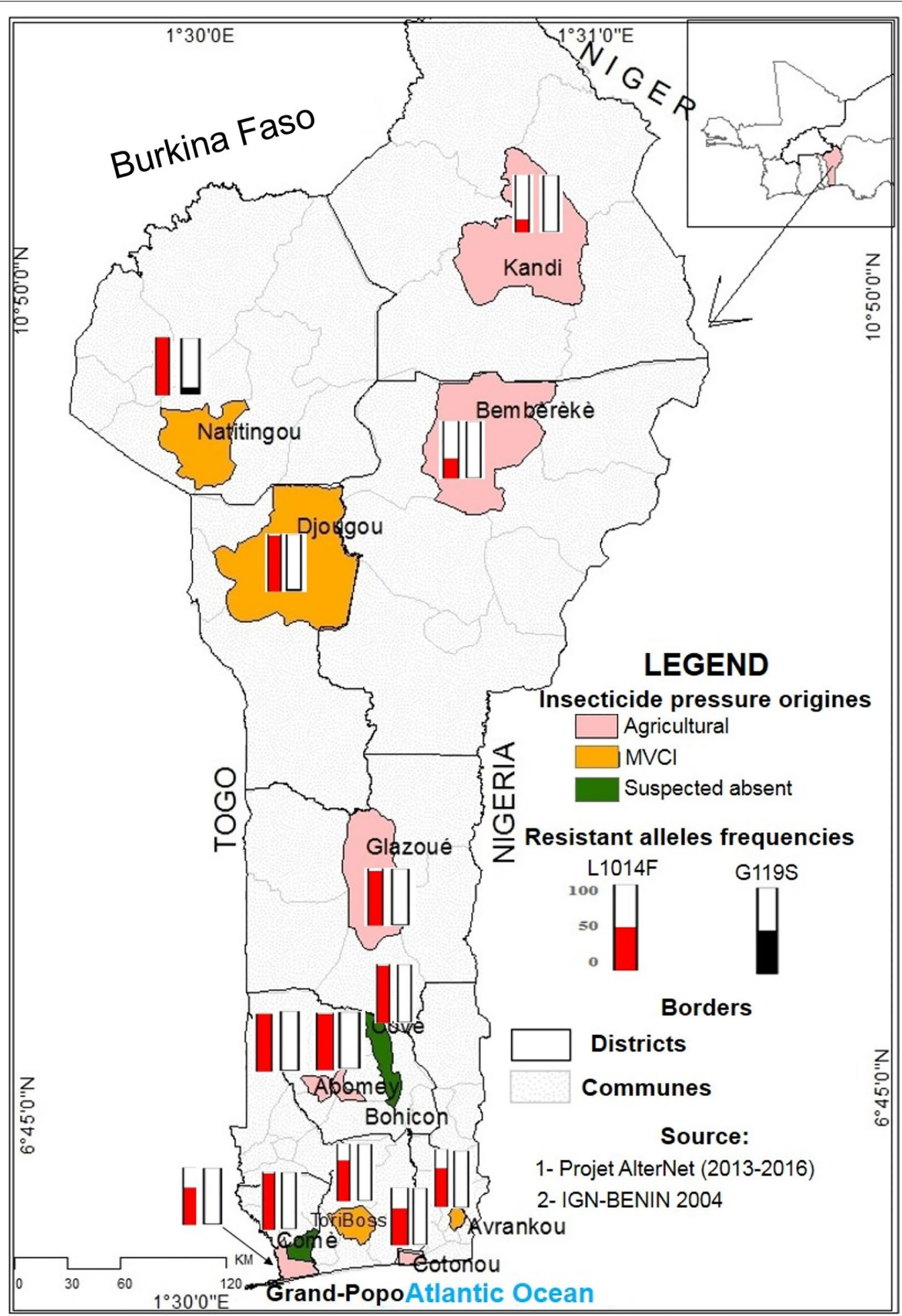

Fig. 1 Map of the sample sites and insecticide resistant allele frequencies (L1014F-VGSC and G119S-ace-1) in natural populations of An. gambiae (s.l.) from of Benin. Abbreviation: MVCl, malaria vector control intervention 
Table 1 List of studied localities and their characteristics

\begin{tabular}{|c|c|c|c|c|c|}
\hline Locality & Environment & Agricultural practice & Agroclimatic zone & Insecticide pressure origin & Reference \\
\hline Cotonou & Urban & Vegetables & Guinean & Agriculture (OCs/OPs/PYRs) & {$[6]$} \\
\hline Grand-Popo & Urban & Vegetables & Guinean & Agriculture (OCs/OPs/PYRs) & Present study \\
\hline Bohicon & Urban & Cereals/Cotton & Soudano-Guinean & Agriculture (OCs/OPs/PYRs) & {$[6]$} \\
\hline Abomey & Urban & Cereals/Cotton & Soudano-Guinean & Agriculture (OCs/OPs/PYRs) & {$[6]$} \\
\hline Glazoue & Urban & Cereals & Soudano-Guinean & Agriculture (OCs/OPs/PYRs) & {$[6]$} \\
\hline Kandi & Rural & Cotton & Soudanian & Agriculture (OCs/OPs/PYRs) & {$[6]$} \\
\hline Bembèrèkè & Rural & Cotton & Soudanian & Agriculture (OCs/OPs/PYRs) & {$[6]$} \\
\hline Tori-Bossito & Rural & Cereals/Fruits & Guinean & MVCI (CXs/PYRs) & {$[6]$} \\
\hline Avrankou & Rural & Cereals & Guinean & MVCI (CXs/PYRs) & Present study \\
\hline Djougou & Urban & Cotton & Soudanian & MVCI (CXs/PYRs) & {$[6]$} \\
\hline Natitingou & Urban & Cotton & Soudanian & MVCI (OPs/CXs/PYRs) & {$[6]$} \\
\hline Comé & Rural & Cereals & Guinean & Average & {$[11]$} \\
\hline Covè & Rural & Cereals & Soudano-Guinean & Average & Present study \\
\hline
\end{tabular}

The collection period was from August to September in 2013, 2014 and 2015

Notes: OCs, PYRs, OPs and CXs correspond to organochlorines, pyrethroids, organophosphates and carbamates insecticides, respectively. MVCI corresponds to areas with reinforced malaria vector control interventions. Agriculture corresponds to areas where exposure to insecticides results mostly from their uses for crop cultures. Average corresponds to areas with limited insecticide uses for domestic agriculture and vector control (low turnover of impregnated bednets, see text)

of 20-25 mosquitoes per tube were exposed to filter papers impregnated with insecticide for $1 \mathrm{~h}$ supplied by the WHO Collaborating Centre, Universiti Sains Malaysia, Penang, Malaysia. After exposure, mosquitoes were transferred to a clean holding tube supplied with $10 \%$ sugar meal and mortality was recorded $24 \mathrm{~h}$ after exposure. Two insecticides were tested: permethrin $(0.75 \%$, a pyrethroid type I) and bendiocarb ( $0.1 \%$ carbamate). According to the WHO recommendations [23], the susceptibility status of each locality was accessed as susceptible, likely resistant and resistant, when observed mortality was $98-100 \%, 90-98 \%$ and less than 90\%, respectively. After exposure to insecticide, survivors and dead mosquitoes were stored in silica gel for further analysis.

\section{Genotyping of L1014F-VGSC and G119S-ace-1}

In An. gambiae (s.l.), the L1014F-VGSC and G119S-ace-1 mutations are the major insecticide target-site resistance alleles for PYRs and CXs resistance, respectively. After exposure to permethrin (PYRs) or bendiocarb (CXs), survivors and dead individuals were genotyped using classical molecular tests [24, 25]. Moreover, 2263 individuals from the 13 localities, unexposed to insecticides, were also genotyped for these mutations to assess resistant alleles frequency dynamics in natural populations.

In populations where a heterozygote deficit was found, the VGSC locus was Sanger-sequenced to confirm VGSC genotypes using the Agd1 and Agd2 primers (293 bp PCR fragment), as described in [22]. We also sequenced (i) two resistant homozygotes An. arabiensis from Djougou (2015); (ii) one resistant homozygote An. arabiensis, one heterozygote and one resistant homozygote $A n$. coluzzii from Bembèrèkè (2015); and (iii) one susceptible homozygote and one heterozygote An. arabiensis, as well as one susceptible homozygote An. coluzzii from Glazoué (2015).

\section{Statistical analyses \\ Data from bioassays}

To test whether the mortality (MORTAL) was impacted by several variables, a generalized linear mixed model (GLMM) was computed as: MORTAL $=$ SELECT + YeAR + Select:Year $+(1 \mid$ Pop $)+\varepsilon$, where Select is a 3-level factor indicating the nature of the insecticide pressure, Pop is a multi-level factor corresponding to the sampled population and YEAR is a 3-level factor corresponding to the year of sampling (Table 1); ":" indicates an interaction between factors, $\varepsilon$ is the error parameter, following a binomial distribution. The PoP factor was computed as a random effect, because each population was exposed to only one kind of insecticide pressure. As bioassays were performed on adults and they were all morphological identified belonging to $A n$. gambiae (s.l.), it was not possible to take into account the Anopheles species independently. The Pop factor thus includes inter-population differences in urbanness, bioclimate and species. The model was simplified as follows: significance of the different fixed terms was tested, starting from the interaction term, using likelihood ratio tests (LRT) corrected for overdispersion [26, 27] as described by Crawley [28]; non-significant terms $(P>0.05)$ were removed. 
Fisher's exact test was used to compare the L1014F$V G S C$ and G119S-ace-1 phenotypic distributions (i.e. number of RR, RS and SS individuals) between dead mosquitoes and survivors, independently in each population, each year and for each species. Sequential Bonferroni correction for multiple testing was used to identify potential false positives [29].

\section{Population genetics analysis}

To analyze the population genetic structure, we used Genepop version 4.2 online (http://genepop.curtin.edu. au). In the 2013-2015 samples, $F_{I S}$ (assessing deviation from the panmictic equilibrium) were estimated independently in each species, each population and each year and $F_{S T}$ (assessing differentiation between populations, i.e. population structure) independently for each year and species (according to Weir \& Cockerham [30]).

To analyze the distribution and long-term dynamics of the resistance allele frequencies, L1014F-VGSC and G119S-ace-1 genotyping data, collected in the same sampling sites between 2006 and 2010 and published in previous studies $[7,11,31-35]$, or collected during this study (2013-2015), were combined. Multinomial loglinear models (multinom function in the R package nnet) were computed independently for each locus as: GENO $=$
Pop + Year + Sp + Pop: Year + Pop: Sp + Year: Sp + Pop:YeAR:Sp $+\varepsilon$, where GENO is a 3-level response variable corresponding to numbers of individuals for each genotype RR, RS and SS, Pop is a multi-level factor corresponding to the sampled population, YEAR is a continuous variable corresponding to the year of sampling (to test for temporal variations) and SP is a 3-level factor corresponding to the species (arabiensis, gambiae or coluzzii); ":" indicates an interaction between variables, $\varepsilon$ is the error parameter, following a multinomial distribution.

These models were simplified as indicated above. When significant YEAR effect or YEAR:POP interactions were detected, thus suggesting inter-annual variations of the genotype distribution, we analyzed each population of each species independently. We tested the significance of the YEAR variable by comparing (using LRT) the models GENO $=$ YEAR $+\varepsilon$ and GENO $=1+\varepsilon$. Sequential Bonferroni correction for multiple testing was used to identify potential false positives [29]. All computations were performed using the R free software (v.3.3.3) [28].

\section{Results}

High permethrin resistance but low bendiocarb resistance In Benin, exposure of An. gambiae (s.l.). populations to insecticides has two main origins: agriculture or vector

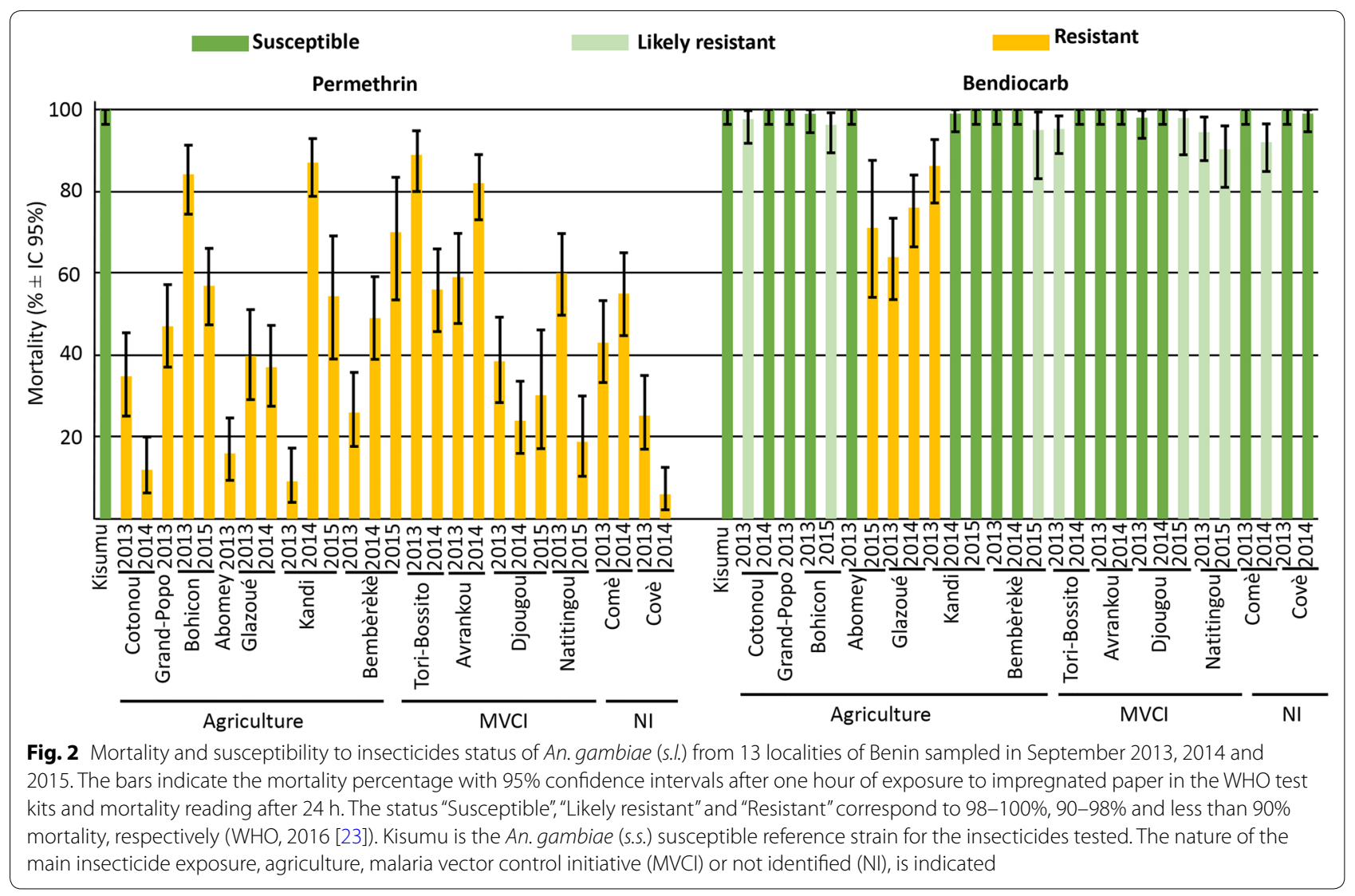


control. During three years, we surveyed 13 populations from Benin, to assess their potential impacts on insecticide resistance dynamics (Table 1, Fig. 1). These localities were chosen because they presented strong contrasts in their main selective pressure. In crop production localities, agricultural usages of insecticides exceed by far the public-health exposure ("Agriculture" localities, Table 1). Other localities, with only limited crop farming, were chosen because vector control ("MVCI" localities, Table 1) is increased through more frequent distribution of impregnated bednets (1-year turnover) and indoor residual spraying in houses, so that public health is the main source of insecticides. Other localities were chosen because they present more limited insecticide usage ("Average" localities, Table 1). Note however, that agricultural usage for domestic production, as well as exposure to impregnated bednets (3-year turnover), are present in all these populations.

To first assess differences in resistance levels, larvae were collected in natural populations, emerged in the laboratory and 3-5-days-old females were exposed to permethrin or bendiocarb at the WHO discriminating doses. Mortality in control groups (i.e. unexposed to insecticides) was consistently $<5 \%$, while the mortality of Kisumu individuals (the susceptible reference strain) was above $99 \%$ for both insecticides. All tested populations showed resistance to permethrin (mortality $<90 \%$ ), but the mortality rate was highly variable between populations and between years (Fig. 2, permethrin).

We found a significant effect of the interaction between the nature of the main insecticide pressures and the collection year on mortality (GLMM, LRT corrected for overdispersion, $P<0.001$; note that a large overdispersion, 3.01, was observed). Therefore, the effect of the nature of insecticide pressures on mortality was analyzed for each year independently. While no significant difference was found in 2013 and 2014 (GLMM, LRT, $P$-value > 0.05; no overdispersion), in 2015 the mortality in populations exposed to insecticides mostly from agriculture origins (Bohicon, Kandi and Bembèrèkè) was significantly higher than in populations exposed mostly to MVCI (Djougou and Natitingou) (GLMM, LRT, $P<0.001$; no overdispersion).

Bendiocarb susceptibility was generally high (Fig. 2, bendiocarb), although some variability was observed between populations and between years. The interaction between the nature of the insecticide pressures and the collection year again showed a significant effect on mortality (GLMM, LRT corrected for overdispersion, $P$-value $<0.001$; moderate overdispersion, 1.46). Analyzing each year independently, we found no significant effect of the nature of insecticide pressures on mortality (GLMM, LRT, $P$-value $>0.05$; no overdispersion).
For each population independently, the dead or surviving larvae after exposure to permethrin or bendiocarb were genotyped for the L1014F-VGSC or G119S-ace-1 mutations, respectively.

After exposure to permethrin, the frequency of L1014F-VGSC mutation was very high in both the dead and surviving individuals for all populations, usually over 0.7 (Additional file 1: Table S1). The L1014F-VGSC phenotypic distributions (i.e. numbers of RR, RS and SS individuals) were, however, significantly different between dead and surviving larvae overall (i.e. pooling all samples) for each year independently (Fisher's exact test on all dead and surviving larvae and all species, $P<0.001$, Additional file 1: Table S3). However, there was no consistent difference between species or between years for any population (ex. for Anopheles arabiensis, we found no phenotypic distribution difference between dead and surviving larvae in 2013, significant differences in 2014, but again none in 2015; Additional file 1: Table S3).

After exposure to bendiocarb, there were overall very few individuals carrying the G119S-ace-1 mutation in both the dead and surviving individuals for all populations and the mutation frequency was usually below 0.05 in dead and usually over 0.2 in surviving larvae (Additional file 1: Table S2). Again, there was no consistent G119S-ace-1 phenotypic distribution difference between the dead and surviving larvae, be it between species or between years for any population (Additional file 1: Table S3).

\section{Heterogeneous resistance allele frequencies in natural populations of An. gambiae (s.l.)}

A total of 2263 individuals collected from the same sites from 2013 to 2015, but unexposed to insecticides (50-60 from each site, Table 2) were then analyzed at the molecular level. We first assessed to which species these individuals belonged and found three Anopheles species, $A n$. coluzzii $(n=1198)$, An. gambiae (s.s.) $(n=782)$ and An. arabiensis $(n=271)$, as well as very rare coluzzii-gambiae (s.s.) hybrids $(n=12)$ (see Table 2). These individuals were then screened for L1014F-VGSC and G119S-ace-1 mutations to assess their frequencies and follow-up their evolution in natural populations.

The L1014F-VGSC mutation was found in all three Anopheles species. However, its frequency was relatively low in An. arabiensis (0-0.3, with an average 0.21 \pm 0.37 , Fig, 3, Table 2), except in Glazoué in 2015, where the frequency was 0.97 (NB: genotype and species were confirmed by Sanger-sequencing three $R R$ individuals). By contrast, the mutation was found in all localities and almost fixed in An. coluzzii and An. gambiae (s.s.), with average frequencies of $0.79 \pm 0.27$ and 0.93 \pm 0.12 , respectively (Fig. 3 , Table 2 ). We then analyzed 
Table 2 Frequencies of L1014F-VGSC and G119S-ace-1 mutations over ten years (2006-2015) in Anopheles gambiae (s.l.) populations of Benin

\begin{tabular}{|c|c|c|c|c|c|c|c|c|c|c|c|c|c|}
\hline \multirow[t]{3}{*}{ Species } & \multirow[t]{3}{*}{ Locality } & \multirow[t]{3}{*}{ Year } & \multicolumn{10}{|c|}{ Insecticide resistance alleles } & \multirow[t]{3}{*}{ Reference } \\
\hline & & & \multicolumn{4}{|c|}{ G119S-ace-1 } & \multirow[t]{2}{*}{$P$-value } & \multicolumn{4}{|c|}{ L1014F-VGSC } & \multirow[t]{2}{*}{$P$-value } & \\
\hline & & & SS & RS & $\mathrm{RR}$ & $f$ & & SS & RS & $\mathrm{RR}$ & $f$ & & \\
\hline \multirow[t]{16}{*}{ An. arabiensis } & \multirow[t]{3}{*}{ Bembèrèkè } & 2013 & 10 & 0 & 0 & 0 & \multirow[t]{3}{*}{ ns } & 7 & 0 & 3 & 0.30 & \multirow[t]{3}{*}{ ns } & Present study \\
\hline & & 2014 & 39 & 0 & 0 & 0 & & 39 & 0 & 0 & 0 & & Present study \\
\hline & & 2015 & 15 & 0 & 0 & 0 & & 14 & 1 & 0 & 0.03 & & Present study \\
\hline & \multirow[t]{3}{*}{ Bohicon } & 2006 & 66 & 0 & 0 & 0 & \multirow[t]{3}{*}{ ns } & 66 & 0 & 0 & 0 & \multirow[t]{3}{*}{ ns } & {$[6]$} \\
\hline & & 2008 & - & - & - & - & & 1 & 4 & 2 & 0.57 & & {$[29]$} \\
\hline & & 2014 & 15 & 0 & 0 & 0 & & 15 & 0 & 0 & 0 & & Present study \\
\hline & \multirow[t]{2}{*}{ Djougou } & 2014 & 1 & 0 & 0 & 0 & \multirow[t]{2}{*}{ ns } & 1 & 0 & 0 & 0 & \multirow[t]{2}{*}{ ns } & Present study \\
\hline & & 2015 & 3 & 0 & 0 & 0 & & 0 & 0 & 3 & 1 & & Present study \\
\hline & \multirow[t]{4}{*}{ Glazoué } & 2007 & 57 & 0 & 0 & 0 & \multirow[t]{4}{*}{ ns } & 57 & 0 & 0 & 0 & $* * *$ & [6] \\
\hline & & 2013 & 36 & 0 & 0 & 0 & & 36 & 0 & 0 & 0 & & Present study \\
\hline & & 2014 & 39 & 0 & 0 & 0 & & 39 & 0 & 0 & 0 & & Present study \\
\hline & & 2015 & 58 & 0 & 0 & 0 & & 2 & 0 & 56 & 0.97 & & Present study \\
\hline & Kandi & 2007 & 32 & 0 & 0 & 0 & ns & 32 & 0 & 0 & 0 & ns & {$[6]$} \\
\hline & & 2013 & 16 & 0 & 0 & 0 & & 13 & 0 & 3 & 0.19 & & Present study \\
\hline & & 2014 & 38 & 0 & 0 & 0 & & 36 & 0 & 2 & 0.05 & & Present study \\
\hline & & 2015 & 1 & 0 & 0 & 0 & & 1 & 0 & 0 & 0 & & Present study \\
\hline An. coluzzii & Abomey & 2006 & 3 & 0 & 0 & 0 & ns & 2 & 1 & 0 & 0.17 & ns & {$[6]$} \\
\hline & & 2013 & 6 & 0 & 0 & 0 & & 0 & 0 & 6 & 1 & & Present study \\
\hline & & 2014 & 50 & 3 & 0 & 0.03 & & 0 & 0 & 53 & 1 & & Present study \\
\hline & & 2015 & 29 & 0 & 0 & 0 & & 0 & 0 & 29 & 1 & & Present study \\
\hline & Avrankou & 2013 & 35 & 2 & 0 & 0 & ns & 5 & 8 & 24 & 0.76 & ns & Present study \\
\hline & & 2014 & 59 & 1 & 0 & 0 & & 3 & 13 & 44 & 0.84 & & Present study \\
\hline & & 2015 & 60 & 0 & 0 & 0 & & 10 & 20 & 30 & 0.67 & & Present study \\
\hline & Bembèrèkè & 2015 & 13 & 0 & 0 & 0 & - & 3 & 7 & 3 & 0.50 & - & Present study \\
\hline & Bohicon & 2006 & 2 & 0 & 0 & 0 & ns & 0 & 0 & 2 & 1 & $* * *(n s)$ & {$[6]$} \\
\hline & & 2008 & - & - & - & - & & 5 & 1 & 1 & 0.21 & & [29] \\
\hline & & 2010 & - & - & - & - & & 1 & 0 & 11 & 0.92 & & [29] \\
\hline & & 2013 & 44 & 0 & 0 & 0 & & 1 & 3 & 40 & 0.94 & & Present study \\
\hline & & 2014 & 28 & 0 & 0 & 0 & & 1 & 3 & 24 & 0.91 & & Present study \\
\hline & & 2015 & 25 & 0 & 0 & 0 & & 0 & 1 & 24 & 0.98 & & Present study \\
\hline & Comè & 2009 & 30 & 0 & 0 & 0 & ns & 1 & 11 & 18 & 0.78 & $*(\mathrm{~ns})$ & [30] \\
\hline & & 2013 & 56 & 0 & 0 & 0 & & 0 & 3 & 53 & 0.97 & & Present study \\
\hline & & 2014 & 51 & 1 & 0 & 0.01 & & 2 & 12 & 37 & 0.84 & & Present study \\
\hline & & 2015 & 60 & 0 & 0 & 0 & & 0 & 7 & 53 & 0.94 & & Present study \\
\hline & Cotonou & 2006 & - & - & - & - & ns & 93 & 140 & 67 & 0.46 & $* * *$ & [31] \\
\hline & & 2007 & 92 & 0 & 0 & 0 & & 3 & 13 & 79 & 0.90 & & {$[6]$} \\
\hline & & 2008 & - & - & - & - & & 3 & 5 & 31 & 0.86 & & [29] \\
\hline & & 2009 & 72 & 0 & 0 & 0 & & 3 & 29 & 97 & 0.86 & & {$[29,30]$} \\
\hline & & 2010 & - & - & - & - & & 0 & 9 & 37 & 0.90 & & [29] \\
\hline
\end{tabular}


Table 2 (continued)

\begin{tabular}{|c|c|c|c|c|c|c|c|c|c|c|c|c|c|}
\hline \multirow[t]{3}{*}{ Species } & \multirow[t]{3}{*}{ Locality } & \multirow[t]{3}{*}{ Year } & \multicolumn{10}{|c|}{ Insecticide resistance alleles } & \multirow[t]{3}{*}{ Reference } \\
\hline & & & \multicolumn{4}{|c|}{ G119S-ace-1 } & \multirow[t]{2}{*}{$P$-value } & \multicolumn{4}{|c|}{ L1014F-VGSC } & \multirow[t]{2}{*}{$P$-value } & \\
\hline & & & SS & RS & $\mathrm{RR}$ & $f$ & & SS & RS & $\mathrm{RR}$ & $f$ & & \\
\hline & & 2013 & 60 & 0 & 0 & 0 & & 0 & 3 & 57 & 0.98 & & Present study \\
\hline & & 2014 & 33 & 3 & 0 & 0.04 & & 0 & 1 & 35 & 0.99 & & Present study \\
\hline & & 2015 & 59 & 0 & 0 & 0 & & 8 & 27 & 24 & 0.64 & & Present study \\
\hline & Covè & 2013 & 58 & 0 & 0 & 0 & ns & 0 & 11 & 47 & 0.91 & ns & Present study \\
\hline & & 2014 & 59 & 0 & 0 & 0 & & 1 & 12 & 46 & 0.88 & & Present study \\
\hline & & 2015 & 59 & 0 & 0 & 0 & & 1 & 2 & 56 & 0.97 & & Present study \\
\hline & Djougou & 2015 & 1 & 0 & 0 & 0 & - & 1 & 0 & 0 & 0 & - & Present study \\
\hline & Glazoué & 2013 & 1 & 0 & 0 & 0 & ns & 0 & 0 & 1 & 1 & ns & Present study \\
\hline & & 2014 & 4 & 0 & 0 & 0 & & 1 & 0 & 3 & 0.75 & & Present study \\
\hline & & 2015 & 1 & 0 & 0 & 0 & & 1 & 0 & 0 & 0 & & Present study \\
\hline & Grand-Popo & 2013 & 56 & 7 & 0 & 0.06 & ns & 0 & 3 & 60 & 0.98 & ns & Present study \\
\hline & & 2014 & 60 & 0 & 0 & 0 & & 1 & 21 & 38 & 0.81 & & Present study \\
\hline & & 2015 & 57 & 1 & 0 & 0.01 & & 11 & 19 & 28 & 0.65 & & Present study \\
\hline & Kandi & 2014 & 2 & 0 & 0 & 0 & - & 1 & 1 & 0 & 0.25 & - & Present study \\
\hline & Tori Bossito & 2007 & 41 & 0 & 0 & 0 & ns & 33 & 8 & 0 & 0.10 & $* * *$ & {$[32]$} \\
\hline & & 2008 & 62 & 0 & 0 & 0 & & 33 & 16 & 13 & 0.34 & & {$[32]$} \\
\hline & & 2009 & - & - & - & - & & 0 & 5 & 25 & 0.92 & & {$[29]$} \\
\hline & & 2010 & - & - & - & - & & 0 & 14 & 16 & 0.77 & & {$[29]$} \\
\hline & & 2013 & 60 & 0 & 0 & 0 & & 0 & 2 & 58 & 0.98 & & Present study \\
\hline & & 2014 & 36 & 0 & 0 & 0 & & 1 & 6 & 29 & 0.89 & & Present study \\
\hline & & 2015 & 58 & 0 & 0 & 0 & & 5 & 24 & 29 & 0.71 & & Present study \\
\hline \multirow[t]{16}{*}{ An. gambiae (s.s.) } & Abomey & 2006 & 52 & 16 & 0 & 0.12 & $* * *$ & 2 & 8 & 58 & 0.91 & ns & {$[6]$} \\
\hline & & 2013 & 47 & 2 & 0 & 0.02 & & 0 & 0 & 49 & 1 & & Present study \\
\hline & & 2014 & 4 & 0 & 0 & 0 & & 0 & 0 & 4 & 1 & & Present study \\
\hline & & 2015 & 30 & 1 & 0 & 0.02 & & 0 & 0 & 31 & 1 & & Present study \\
\hline & Avrankou & 2013 & 22 & 1 & 0 & 0.02 & - & 3 & 5 & 15 & 1 & - & Present study \\
\hline & Bembèrèkè & 2007 & 57 & 5 & 0 & 0.04 & * (ns) & 5 & 15 & 42 & 0.80 & ns & {$[6]$} \\
\hline & & 2013 & 47 & 0 & 0 & 0 & & 0 & 1 & 46 & 0.99 & & Present study \\
\hline & & 2014 & 21 & 0 & 0 & 0 & & 4 & 0 & 17 & 0.81 & & Present study \\
\hline & & 2015 & 19 & 0 & 0 & 0 & & 6 & 8 & 5 & 0.47 & & Present study \\
\hline & Bohicon & 2006 & 2 & 1 & 0 & 0.17 & ns & 0 & 1 & 3 & 0.88 & ns & {$[6]$} \\
\hline & & 2008 & - & - & - & - & & 3 & 6 & 16 & 0.76 & & {$[29]$} \\
\hline & & 2009 & - & - & - & - & & 0 & 4 & 41 & 0.96 & & {$[29]$} \\
\hline & & 2010 & - & - & - & - & & 0 & 7 & 26 & 0.89 & & [29] \\
\hline & & 2013 & 15 & 0 & 0 & 0 & & 0 & 0 & 15 & 1 & & Present study \\
\hline & & 2014 & 14 & 0 & 0 & 0 & & 0 & 1 & 13 & 0.96 & & Present study \\
\hline & & 2015 & 35 & 0 & 0 & 0 & & 0 & 2 & 33 & 0.97 & & Present study \\
\hline
\end{tabular}


Table 2 (continued)

\begin{tabular}{|c|c|c|c|c|c|c|c|c|c|c|c|c|c|}
\hline \multirow[t]{3}{*}{ Species } & \multirow[t]{3}{*}{ Locality } & \multirow[t]{3}{*}{ Year } & \multicolumn{10}{|c|}{ Insecticide resistance alleles } & \multirow[t]{3}{*}{ Reference } \\
\hline & & & \multicolumn{4}{|c|}{ G119S-ace-1 } & \multirow[t]{2}{*}{$P$-value } & \multicolumn{4}{|c|}{ L1014F-VGSC } & \multirow[t]{2}{*}{$P$-value } & \\
\hline & & & SS & RS & $\mathrm{RR}$ & $f$ & & SS & RS & $\mathrm{RR}$ & $f$ & & \\
\hline & Comè & 2013 & 4 & 0 & 0 & 0 & - & 0 & 0 & 4 & 1.00 & - & Present study \\
\hline & Covè & 2014 & 1 & 0 & 0 & 0 & ns & 0 & 0 & 1 & 1.00 & ns & Present study \\
\hline & & 2015 & 1 & 0 & 0 & 0 & & 0 & 0 & 1 & 1.00 & & Present study \\
\hline & Djougou & 2007 & 39 & 3 & 0 & 0.04 & ns & 1 & 10 & 31 & 0.86 & ns & {$[6]$} \\
\hline & & 2013 & 60 & 0 & 0 & 0 & & 0 & 3 & 57 & 0.98 & & Present study \\
\hline & & 2014 & 55 & 0 & 0 & 0 & & 1 & 2 & 52 & 0.96 & & Present study \\
\hline & & 2015 & 51 & 4 & 0 & 0.04 & & 1 & 0 & 54 & 0.98 & & Present study \\
\hline & Glazoué & 2007 & 5 & 0 & 0 & 0 & ns & 4 & 1 & 0 & 0.10 & ns & {$[6]$} \\
\hline & & 2013 & 23 & 0 & 0 & 0 & & 0 & 0 & 23 & 1 & & Present study \\
\hline & & 2014 & 14 & 1 & 0 & 0.03 & & 0 & 1 & 14 & 0.97 & & Present study \\
\hline & & 2015 & 0 & 1 & 0 & 0.50 & & 0 & 0 & 1 & 1 & & Present study \\
\hline & Kandi & 2007 & 30 & 0 & 0 & 0 & ns & 7 & 8 & 15 & 0.63 & $* * *$ & {$[6]$} \\
\hline & & 2009 & 90 & 3 & 0 & 0.02 & & 80 & 104 & 90 & 0.52 & & [11] \\
\hline & & 2013 & 44 & 0 & 0 & 0 & & 3 & 0 & 41 & 0.93 & & Present study \\
\hline & & 2014 & 20 & 0 & 0 & 0 & & 4 & 0 & 16 & 0.80 & & Present study \\
\hline & & 2015 & 58 & 0 & 0 & 0 & & 13 & 0 & 45 & 0.78 & & Present study \\
\hline & Natitingou & 2007 & 47 & 1 & 0 & 0.01 & ns & 1 & 13 & 47 & 0.88 & $* * *$ & {$[6]$} \\
\hline & & 2012 & - & - & - & - & & 33 & 11 & 0 & 0.13 & & [33] \\
\hline & & 2013 & 60 & 0 & 0 & 0 & & 0 & 0 & 60 & 1 & & Present study \\
\hline & & 2014 & 46 & 3 & 0 & 0.03 & & 0 & 0 & 49 & 1 & & Present study \\
\hline & & 2015 & 46 & 12 & 0 & 0.10 & & 0 & 0 & 58 & 1 & & Present study \\
\hline & Tori Bossito & 2007 & 144 & 2 & 0 & 0.01 & ns & 74 & 62 & 10 & 0.28 & $* * *$ & [32] \\
\hline & & 2008 & 71 & 0 & 0 & 0 & & 43 & 32 & 32 & 0.45 & & {$[29,32]$} \\
\hline & & 2009 & - & - & - & - & & 0 & 5 & 20 & 0.90 & & [29] \\
\hline & & 2010 & - & - & - & - & & 1 & 5 & 5 & 0.68 & & [29] \\
\hline & & 2014 & 20 & 0 & 0 & 0 & & 0 & 0 & 20 & 1 & & Present study \\
\hline \multirow{5}{*}{$\begin{array}{l}\text { An. coluzii } \times \text { An. gam- } \\
\text { biae (s.s.) hybrids }\end{array}$} & Abomey & 2013 & 5 & 0 & 0 & 0 & - & 0 & 0 & 5 & 1 & - & Present study \\
\hline & Bohicon & 2013 & 1 & 0 & 0 & 0 & - & 0 & 0 & 1 & 1 & - & Present study \\
\hline & Bembèrèkè & 2015 & 1 & 0 & 0 & 0 & - & 0 & 1 & 0 & 0.5 & - & Present study \\
\hline & Djougou & 2015 & 1 & 0 & 0 & 0 & - & 0 & 0 & 1 & 1 & - & Present study \\
\hline & Tori Bossito & 2014 & 4 & 0 & 0 & 0 & - & 0 & 0 & 4 & 1 & - & Present study \\
\hline
\end{tabular}

Notes: For each species of the complex, the year of sampling and the number of individuals per genotype are indicated for both resistance mutations, as well as the source of the data. For each resistance mutation, the frequency of the resistant allele is calculated for each sample $(f)$ and the P-value indicates whether there is a significant interannual variation (multinomial log-linear model, YEAR effect, see "Methods"): ns, $P>0.05,{ }^{*} P \leq 0.05,{ }^{* *} P \leq 0.01$, *** $P \leq 0.001$ (when the Bonferroni correction suggests that the significant $P$-value is probably a false positive, ns is indicated within brackets). " $-"$ is indicated when no data were available 


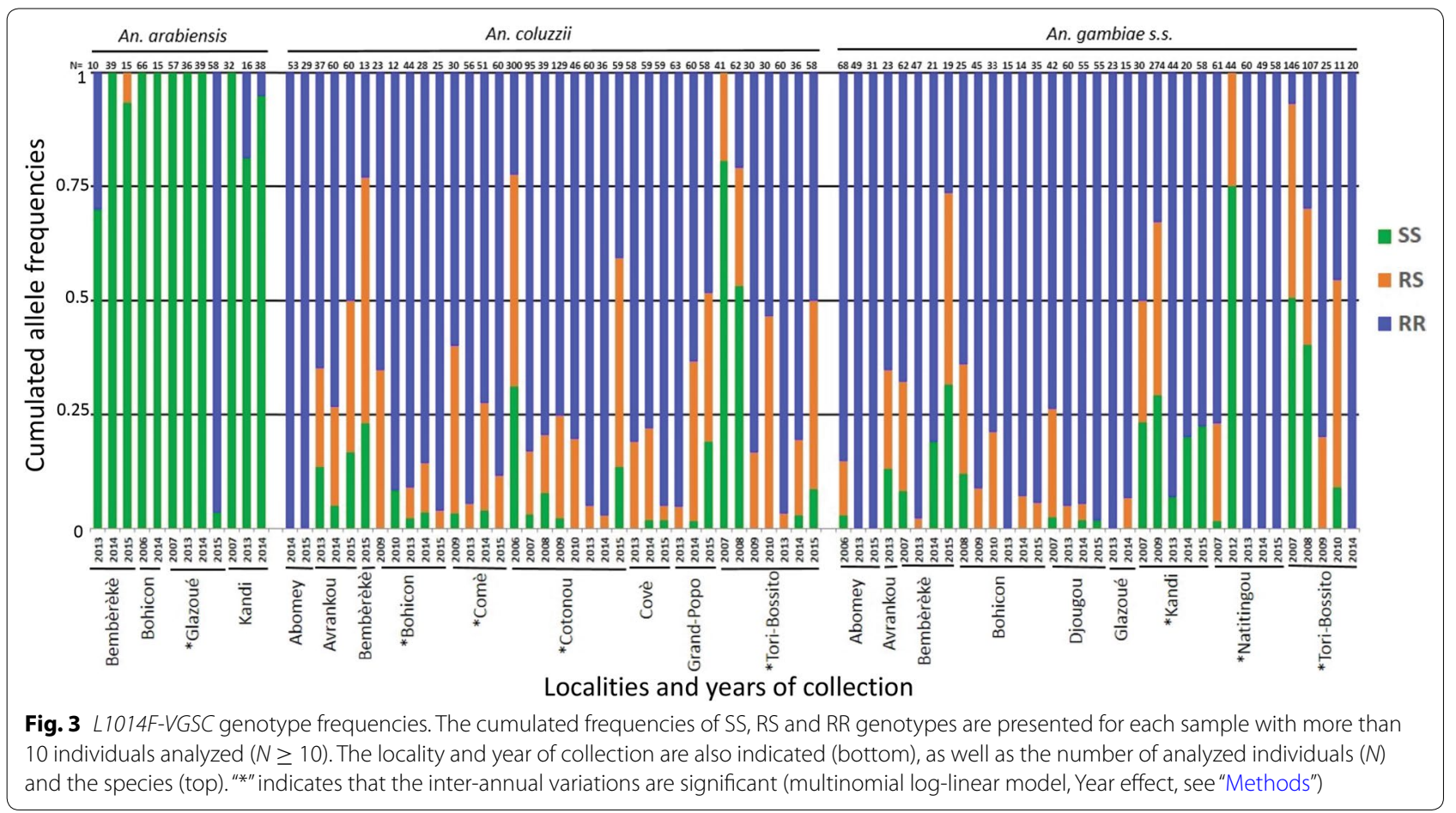

the population genetic structure (using $F$ statistics in the sense of Weir \& Cockerham [30]) and found that most populations were not different from a panmictic equilibrium (most $F_{I S}$ were not statistically different from 0 , Table 3), with significant differentiation between populations $\left(F_{S T}<0.2\right)$, that could reach high values as in 2015 (Table 3).

The same samples were also screened for the G119Sace-1 mutation. A total of 271 An. arabiensis individuals carried the mutation; it was found in some populations for An. coluzzii and An. gambiae (s.s.), only in heterozygotes (Fig. 4). Frequencies were low (between 0-0.1, Table 2) and the mutation was often not detected in overall sampling years in some populations (Fig. 4). Due to the rarity of the mutation, most populations were SS monomorphic, which generally prevented the analysis of the $F_{I S}$ (Tables 2,3). Similarly, only low differentiation was found between populations $\left(F_{S T}<0.1\right.$, Table 3).

\section{Over ten years in Benin, $k d r$ fluctuated at generally high frequencies, while ace- $7^{R}$ remained rare}

Adding data published from 2006 to 2010 in previous studies to the 2013-2015 new data, we analysed the distribution and long-term dynamics of the resistance allele frequencies, L1014F-VGSC and G119S-ace-1.

For L1014F-VGSC, multinomial log-linear models showed no significant POP:YEAR:SP interaction (LRT,
$P$-value $>0.05)$, although all Pop:Year, Pop:Sp or YEAR:SP interactions were significant (LRT, $P$-value < 0.001). We thus analysed the YeAR effect independently in each species and population (Table 2, Fig. 3). In $A n$. arabiensis, only Glaouzé showed significant variations in resistance allele frequencies over the years, with a sharp increase in 2015 ( $P$-value $<0.001$, Table 2); in $A n$. coluzzii, significant variations were found in Cotonou and Tori Bossito (with no clear trend, but slight and recent reductions after years at high frequencies) and tendencies to increase in Bohicon and Comè that did not, however, pass correction for multi-testing; in An. gambiae (s.s.), significant variations were found in Kandi (no clear trend, but a slight and recent reduction after years at high frequencies) and Naititingou and Tori Bossito (increases) (Table 2).

For G119S-ace-1, multinomial log-linear models showed no significant Pop:Year:Sp, POP:YeAR, POP:Sp or YeAR:SP interactions (LRT, $P$-value $>0.05$ ). Significant effects were, however, found for the simple factors PoP (LRT, $P$-value $<0.001$ ), SP (LRT, $P$-value $<0.01$ ) and YeAR (LRT, $P$-value $<0.05$ ). We again analyzed the YeAR effect independently in each species and population (Table 2, Fig. 4). Unsurprisingly considering the low mutation frequencies, we found no significant variations in resistance allele frequencies over the years, except in An. gambiae (s.s.) from Abomey, with no clear trend (Table 2). 
Table 3 Population genetics analyses at VGSC and ace-1 locus

\begin{tabular}{|c|c|c|c|c|c|c|c|c|c|c|c|c|c|}
\hline \multirow[t]{3}{*}{ Species } & \multirow[t]{3}{*}{ Population } & \multicolumn{6}{|l|}{ ace-1 } & \multicolumn{6}{|l|}{ VGSC } \\
\hline & & \multicolumn{2}{|l|}{2013} & \multicolumn{2}{|l|}{2014} & \multicolumn{2}{|l|}{2015} & \multicolumn{2}{|l|}{2013} & \multicolumn{2}{|l|}{2014} & \multicolumn{2}{|l|}{2015} \\
\hline & & \multicolumn{2}{|l|}{$F_{15}$} & \multicolumn{2}{|l|}{$F_{15}$} & \multicolumn{2}{|l|}{$F_{15}$} & \multicolumn{2}{|l|}{$F_{15}$} & \multicolumn{2}{|l|}{$F_{15}$} & \multicolumn{2}{|l|}{$F_{15}$} \\
\hline \multirow[t]{6}{*}{ An. arabiensis } & Bèmbèrèkè & - & & - & & - & & 1 & $* *$ & - & & - & \\
\hline & Bohicon & - & & - & & - & & - & & - & & - & \\
\hline & Djougou & - & & - & & - & & - & & - & & - & \\
\hline & Glazoué & - & & - & & - & & - & & - & & 1 & $* * *$ \\
\hline & Kandi & - & & - & & - & & 1 & $* * *$ & 1 & $* * *$ & - & \\
\hline & $F_{S T}$ & $F_{S T}$ & - & $F_{S T}$ & - & $F_{S T}$ & - & $F_{S T}$ & $0.211^{* *}$ & $F_{S T}$ & $0.011 \mathrm{~ns}$ & $F_{S T}$ & $0.917^{* * *}$ \\
\hline \multirow[t]{13}{*}{ An. coluzzii } & Abomey & - & & -0.02 & ns & - & & - & & - & & - & \\
\hline & Avrankou & -0.014 & ns & - & & - & & 0.432 & $*$ & 0.195 & ns & 0.258 & ns \\
\hline & Bèmbèrèkè & - & & - & & - & & - & & - & & -0.037 & ns \\
\hline & Bohicon & - & & - & & - & & 0.38 & ns & 0.357 & ns & - & \\
\hline & Comè & - & & - & & - & & -0.019 & ns & 0.12 & ns & -0.054 & ns \\
\hline & Cotonou & - & & -0.03 & ns & - & & -0.017 & ns & - & & 0.021 & ns \\
\hline & Covè & - & & - & & - & & -0.097 & ns & 0.036 & ns & 0.489 & ns \\
\hline & Djougou & - & & - & & - & & - & & - & & - & \\
\hline & Glazoué & - & & - & & - & & - & & 1 & ns & - & \\
\hline & Grand-Popo & -0.051 & ns & - & & - & & -0.016 & ns & -0.121 & ns & 0.291 & * \\
\hline & Kandi & - & & - & & - & & - & & - & & - & \\
\hline & Tori-Bossito & - & & - & & - & & -0.009 & ns & 0.17 & ns & 0.010 & ns \\
\hline & $F_{S T}$ & $F_{S T}$ & $0.033^{* * *}$ & $F_{S T}$ & $0.008 \mathrm{~ns}$ & $F_{S T}$ & - & $F_{S T}$ & $0.078^{* * *}$ & $F_{S T}$ & $0.058^{* * *}$ & $F_{S T}$ & $0.165^{* * *}$ \\
\hline \multirow[t]{12}{*}{ An. gambiae (s.s.) } & Abomey & -0.01 & ns & - & & - & & - & & - & & - & \\
\hline & Avrankou & - & & - & & - & & 0.421 & ns & - & & - & \\
\hline & Bèmbèrèkè & - & & - & & - & & - & & 1 & $* * *$ & 0.182 & ns \\
\hline & Bohicon & - & & - & & - & & - & & - & & -0.015 & ns \\
\hline & Comè & - & & - & & - & & - & & - & & - & \\
\hline & Covè & - & & - & & - & & - & & - & & - & \\
\hline & Djougou & - & & - & & -0.029 & ns & -0.017 & ns & 0.488 & ns & 1 & $* *$ \\
\hline & Glazoué & - & & - & & - & & - & & - & & - & \\
\hline & Kandi & - & & - & & - & & 1 & ns & 1 & $* * *$ & 1 & $* * *$ \\
\hline & Natitingou & - & & -0.021 & ns & 0.036 & ns & - & & - & & - & \\
\hline & Tori-Bossito & - & & - & & - & & - & & - & & - & \\
\hline & $F_{S T}$ & $F_{S T}$ & - & $F_{S T}$ & $0.002 \mathrm{~ns}$ & $F_{S T}$ & $0.082^{* * *}$ & $F_{S T}$ & $0.119^{* * *}$ & $F_{S T}$ & $0.082^{* *}$ & $F_{S T}$ & $0.660^{* *}$ \\
\hline
\end{tabular}

Notes: $F_{I S}$ is an estimation of deviation from the panmictic equilibrium in each species, each population and each year. $F_{S T}$ is an estimation of differentiation between populations independently for each year and species (tested using Genepop option 3.3). ns, $P>0.05,{ }^{*} P \leq 0.05, * * P \leq 0.01, * * * P \leq 0.001$ ). " ${ }^{* \prime}$ is indicated when no data are available

\section{Discussion}

Environmental variations induced by insecticides used against crop pests or vectors pathogens have been a strong selection pressure on vector populations. To understand how it affects these populations over time at both the phenotypic and genotypic levels, we investigated the susceptibility of An. gambiae (s.l.) to insecticides (permethrin and bendiocarb) and the distribution of the resistance target-site mutations (L1014F-VGSC and G119S-ace-1) in different agroclimatic zones, with different insecticide practices, across Benin. Indeed, climate and insecticide usage are key parameters in understanding the evolutionary dynamics of resistance genes.

\section{Pyrethroids resistance is frequent in all vector populations from Benin, driven by vector control and agriculture}

One of the key questions of our study was to assess whether the origin of the insecticide pressures impacted the spread of resistance in vector populations from Benin.

We showed that all tested $A n$. gambiae (s.l.) populations were consistently and often highly resistant to 

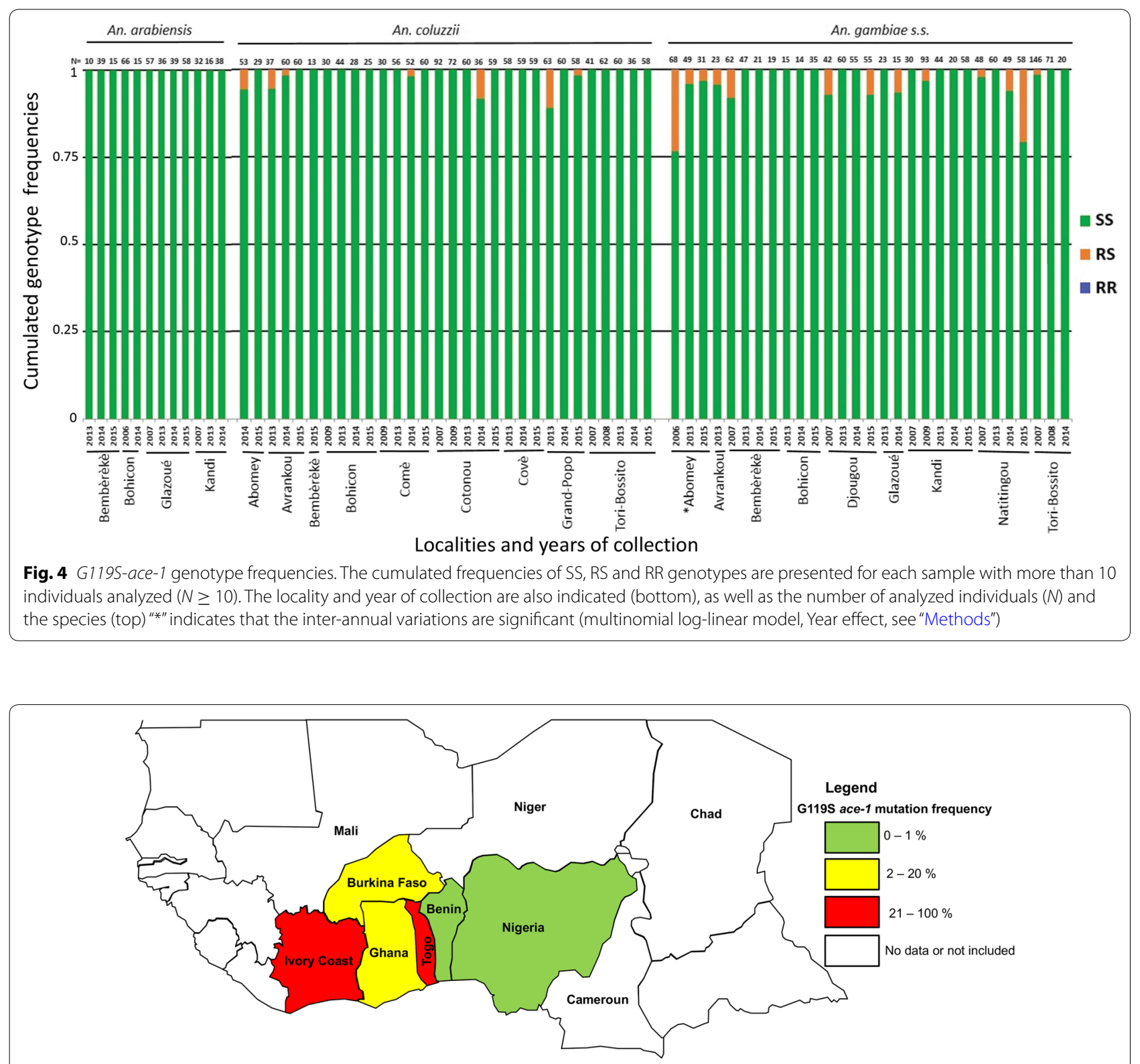

Fig. 5 West-Africa map and G119S ace-1 mutation frequency in natural populations of Anopheles gambiae (s.l.). Data were extracted from this study (Benin, 0.0095\%), Assogba et al. (2018) [56] (Togo, 88.28\%), Oduola et al. (2012) [57] (Nigeria, 0\%), Essandoh et al. (2013) [58] (Ghana, 16.448\%), Alou et al. (2010) [59] (Ivory Coast, 31.25\%) and Dabiré et al. (2014) [60] (Burkina Faso, 18.54\%)

permethrin over the three consecutive years of our survey (Fig. 2, permethrin), which suggests that these mosquito populations are exposed to strong and pervasive pyrethroid insecticide pressures. Despite the difficulties of gathering information on the local treatment practices, we were able to compare populations exposed mainly to vector control treatments (MVCI) with populations exposed mainly to insecticides used for agriculture. As previous studies in Burkina Faso $[2,11]$, we found high PYR resistance in both kinds of localities, which strongly suggests that both practices are sources of selective pressure in Benin. There was, however, a high heterogeneity between populations, even under the same kind of treatment practices. This might be linked to the urban-ness or the bioclimate of the localities, although the number of populations investigated remains low and prevents definitive conclusions. We nevertheless found that, at least in 2015, MVCI resulted in significantly higher resistance levels than exposure to agriculture insecticides (Fig. 2). 
In line with these observations at the phenotypic level, we also found high frequencies of the 1014F-VGSC mutation in the 2263 screened individuals from 13 localities over three years (Table 2, Fig. 3), confirming the tendency observed in previous studies [36] (see Table 2) or more recent years in southern Benin [37]. While the mutation frequency was high (and sometimes fixed) in both dead and survivor mosquitoes after $24 \mathrm{~h}$ exposure to permethrin (Additional file 1: Table S1), there were significant differences that tend to confirm the mutation adaptive advantage (Additional file 1: Table S3). Note however, that metabolic insecticide resistance mechanisms (e.g. P450-monooxigenase) have also been shown by previous studies to contribute to pyrethroid resistance in Benin $[36,38]$.

However, we show in this study that the distribution of the mutation in the different species of the An. gambiae (s.l.) complex is heterogeneous: while we found it in An. coluzzii, An. gambiae (s.s.), as well as in An. arabiensis, its frequency was much lower in the latter and higher in An. gambiae than in An. coluzzii (Fig. 3). This probably results from their ecological differences: while $A n$. arabiensis is described as more zoophilic, exophagic and exophilic in savannah and sparse woodland, albeit with a high plasticity depending on the studies, including breeding in rice fields, An. coluzzii and An. gambiae (s.s.) are described as more anthropophilic, endophagic and endophilic (An. gambiae more so than An. coluzzii), again with variations $[39,40]$. As such, An. gambiae (s.s.) and An. coluzzii (to a slightly lesser extent) would be more exposed than An. arabiensis to pyrethroids currently used in LLINs campaigns, which could explain the differences in L1014F-VGSC frequencies. Consequently, the higher prevalence of An. arabiensis in most populations exposed only to agriculture insecticides rather than MVCI (ex. Kandi, Glazoué, Bohicon and Bembèrèkè) could explain, at least in part, the higher mortality observed in bioassays (Figs. 2, 3).

Our study thus supports the notion that the type of insecticide exposure and the Anopheles species composition interact to explain the observed pyrethroid resistance levels in natural populations.

\section{L1014F-VGSC frequency tends to increase in Benin}

To assess the dynamics of pyrethroid resistance, we combined data published in previous studies (2006-2010) $[7,11,31-35]$ with the data collected for this study (2013-2015) and analyzed the long-term dynamics of the resistance allele frequency. The number of individuals analyzed was variable (Table 2), particularly when taking the species into account, so that the statistical power of our analyses was not optimal. Consequently, while there seems to be a general trend towards an increase in L1014F-VGSC frequency (Table 2, Fig. 3), we only found significant inter-annual variations for some of the populations, but in all three species (Fig. 3). This trend seems to persist in more recent samples (although the authors give regional rather than population-level data [37]). This persistence at high frequencies, despite its fitness costs, indicates that the resistance allele is still under selection (even if metabolic resistance mechanisms are also present $[36,38])$. A similar situation has also been reported in $A n$. gambiae (s.l.) populations from Burkina Faso, where over a ten-year period (between 1999 and 2009), the frequency of L1014F-VGSC mutation increased from 0.2 to 0.8 [41]. In this case, as in a study carried out on vegetable farming sites in southern of Benin [42], indirect evidence suggests that residues of PYRs/OCs in soils might select the L1014F-VGSC mutation, so that agricultural intensification would be one cause for these increases [41]. However, as shown above, vector control interventions are also responsible: a study in Mali indeed suggested that the increasing L1014F-VGSC frequency in An. coluzzii was due to the intensive use of long-lasting insecticidal nets [43]. Our results on the sharp increase in frequency L1014F-VGSC following the distribution of impregnated mosquito nets may reflect the same impact. It is important to note however, that significant decreases were also found in some populations (Table 2, Fig. 3): this shows that, despite the global trends, the local practices, both in agriculture and vector control, can rapidly impact the dynamics of the pyrethroid resistance allele. This heterogeneity in the practices also probably explains the high L1014F-VGSC frequency heterogeneities observed between populations (Additional file 1: Table S1). Overall, our study highlights the finesse of the data required to precisely follow, understand and predict resistance dynamics, but also encourages further investigations to acquire this knowledge as it shows that PYR resistance is not irreversible.

Moreover, this increase of PYR resistance mechanism may affect malaria parasite development within An. gambiae (s.l.) and thereby impact on malaria transmission. However, few studies have shown that the resistant vectors carrying L1014F-VGSC mutation increase the prevalence in oocysts and sporozoites development [44-46]. Moreover, studies designed to assess whether resistance has an impact on current vector control tools (LLINs and IRS), have shown the decrease of their effectiveness to kill resistant vectors [47-50]. Consequently, insecticide resistance could potentially maintain residual malaria transmission and jeopardize malaria elimination in many places of sub-Saharan Africa. 
Resistance to carbamates and organophosphates remains low in Benin, despite high prevalence in neighbouring countries

From 2013 to 2015, bendiocarb resistance of An. gambiae (s.l.) remained generally low in Benin, with very few localities showing less than 90\% mortalities (Abomey, Glazoué and Kandi, Fig. 2, bendiocarb). Although all these localities are mostly exposed to agriculture insecticides rather than MVCI, the nature of insecticide pressures had no statistically significant effect. Note however, that the low resistance levels largely impeded the statistical power of the analyses.

Very few $A n$. arabiensis surviving mosquitoes were obtained in bioassays, except in Glazoué and Cotonou and none carried the G119S mutation (Additional file 1: Table S2). This might signal a lack of adjustment for the bendiocarb diagnostic doses used in the WHO susceptibility tests for this species. It could also be due to the presence of metabolic resistance mechanisms like esterases, which have been described in An. gambiae from Burkina Faso, Ivory Coast, or Mayotte Island, Comoros [51-53]. There were only few survivors in bioassays for An. coluzzii and An. gambiae (s.s.) (Additional file 1: Table S2), with a higher, though still low, frequency of the G119S mutation (Fig. 4). Again, most surviving individuals did not carry this mutation, which could indicate the selection of other resistance mechanisms, such as detoxification.

When pooling data published in previous studies (2006-2010) with the new ones (2263 samples from 2013 to 2015), we confirmed that, although present in most, the mutation remains very rare in natural populations (Fig. 4) with no apparent geographical structure (Table 3). This is actually surprising as many surrounding countries display much higher G119S frequencies (Fig. 5), with no obvious reasons to explain the differences.

However, these results, high susceptibility and very low frequency of G119S-ace-1 in vector populations, provide strong support for the proposed strategy of using CXs/ OPs insecticides as an alternative to PYRs for vector control, at least in Benin. The pervasive and increasing PYR resistance in An. gambiae (s.l.) African populations indeed encouraged a switch from PYRs to CXs/OPs (or mixed applications) in several sub-Saharan African countries to preserve vector control effectiveness, with the support of the American President's Malaria Initiative [10] and the National Malaria Control Programme (including Benin [54]). While this strategy appears interesting for short- or even medium terms, resistance will eventually spread, as the G119S-ace-1 mutation is already present in vector populations and will be selected. A tight monitoring of the resistance markers is thus mandatory from the start, to prevent as long as possible and then try to limit the spread. Again, our study over ten years shows strong fluctuations, with detection of the mutation in some localities (Fig. 4, Table 2). This again suggests that a smart use of the insecticides could be implemented to moderate the selective pressure to slow the mutation spread and to withdraw insecticides in time to benefit from the selective cost associated with G119S-ace-1 $[22,55]$. It will in any case require fine monitoring and anticipation from the different actors involved in vector control, scientists and programme managers, to prevent repeating the previous decisions that led to the PYR resistance situation.

\section{Conclusions}

Our results confirm both the high prevalence of PYR resistance and the potential of CXs/OPs as short- to medium term alternatives in Benin. They also underline the need for regular resistance monitoring and informed management in their usage, as the G119S-ace-1 mutation is already present in Benin and surrounding countries. Unwise usage of CXs/OPs would rapidly lead to its spread, which would jeopardize PYR-resistant Anopheles control.

\section{Supplementary information}

Supplementary information accompanies this paper at https://doi. org/10.1186/s13071-020-4006-6.

Additional file 1: Table S1. L1014F-VGSC allele frequency in survivor and dead Anopheles gambiae (s.l.) after 1-hour exposure to permethrin insecticide. Table S2. G119S-ace-1 allele frequency in survivor and dead of Anopheles gambiae (s.l.) after 1-hour exposure to bendiocarb insecticide. Table S3. P-values of Fisher's exact tests comparing the phenotypic distributions (i.e. numbers of SS, RS and RR individuals) between dead and surviving mosquitoes for the L1014F-VGSC and G119S-ace-1 mutations, after exposure respectively to permethrin and bendiocarb insecticides. $P$-values $<0.05$ are bolded when still significant after sequential Bonferroni correction, italicized otherwise. NB: only populations where more than one phenotype was found are considered here.

\section{Abbreviations}

s.l.: sensu lato; s.s.: sensu stricto; RR: homozygote-resistant; RS: heterozygote; SS: homozygote susceptible; PYRs: pyrethroids; CXs: carbamates; OPs: organophosphates; L1014F-VGSC: mutation leucine by phenylalanine at 1014 position in voltage-gated sodium channel protein; G119S-ace-1: mutation glycine by serine at position 119 in acetylcholinesterase 1 enzyme; GLMM: generalized linear mixed model; LRT: likelihood ratio tests; $F_{i s}$ : proportion of the variance in the subpopulation contained in an individual; $F_{S T}$ : proportion of the total genetic variance contained in a subpopulation (the $S$ subscript) relative to the total genetic variance (the T subscript); $\mathrm{MOH}$ : Ministry of Health; NMCP: National Malaria Control Programme; GPIRM: Global Plan for Insecticide Resistance Management; MVCl: Malaria Vector Control Intervention; WHO: World Health Organization.

\section{Acknowledgements}

We would like to thank Andre S. Sominahouin and Marcellin H. Assogba for mapping these data. BSA was supported by Institut pour la Recherche et le Développement (IRD) PhD fellowship during data collection and by MARCAD/DELTAS Africa Initiative DL-15-010/African Academic of Sciences Postdoc fellowship during the writing of this paper. This work was funded 
partly by French ANR programme (project "AlterNET" SOC \& ENV 2013-2015, ANR-12-SENV-0003). The sequences used in this study were generated by the technical facilities of the Environmental Genomic Platform of the LabEx Centre "Méditerranéen Environnement Biodiversité" and the GenSeq ISEM Platform.

\section{Authors' contributions}

BSA, PL and MW contributed to the conception and design of the experiments. BSA, PM and SU contributed to the data acquisition. BSA, NP, PL and MW contributed to the analysis of the data. BSA, PL and MW drafted the article and LSBM, PL and MM revised it. All authors read and approved the final manuscript.

\section{Funding}

BSA was supported by Institut pour la Recherche et le Développement (IRD) PhD fellowship during data collection and by MARCAD/DELTAS Africa Initiative DL-15-010/African Academic of Sciences Postdoctoral fellowship during the writing of this paper. This study was funded partly by French ANR Programme (project"AlterNET" SOC \& ENV 2013-2015, ANR-12-SENV-0003). The funders did not have any role in the design, collection, analysis and interpretation of data and writing of this manuscript.

\section{Availability of data and materials}

All data generated or analysed during this study are included in this published article and its additional file.

\section{Ethics approval and consent to participate}

Not applicable.

\section{Consent for publication}

Not applicable.

\section{Competing interests}

The authors declare that they have no competing interests.

\section{Author details}

${ }^{1}$ Institut des Sciences de l'Evolution de Montpellier (ISEM), UMR CNRS-IRDEPHE-Université de Montpellier, Place Eugène Bataillon, 34095 Montpellier, France. ${ }^{2}$ Faculté des Sciences et Techniques, Laboratoire de Biologie et de Typage Moléculaire en Microbiologie, Université d'Abomey Calavi, 05 BP 1604, Cotonou, Benin. ${ }^{3}$ Institut Régional de Santé Publique, Université d'Abomey Calavi, 01 BP 918, Cotonou, Benin. ${ }^{4}$ Disease Control and Elimination Department, Medical Research Council, Unit The Gambia at London School of Hygiene and Tropical Medicine, Fajara, Gambia.

Received: 3 December 2019 Accepted: 3 March 2020

Published online: 14 March 2020

\section{References}

1. WHO. World malaria report 2018. Geneva: World Health Organization; 2018.

2. Tokponnon FT, Aholoukpe B, Denon EY, Gnanguenon V, Bokossa A, N'guessan $\mathrm{R}$, et al. Evaluation of the coverage and effective use rate of long-lasting insecticidal nets after nation-wide scale up of their distribution in Benin. Parasit Vectors. 2013;6:265.

3. Hemingway J, Ranson H, Magill A, Kolaczinski J, Fornadel C, Gimnig J, et al. Averting a malaria disaster: will insecticide resistance derail malaria control? Lancet. 2016;387:1785-8.

4. Ranson $\mathrm{H}$, Lissenden N. Insecticide resistance in African Anopheles mosquitoes: a worsening situation that needs urgent action to maintain malaria control. Trends Parasitol. 2016;32:187-96.

5. Alout H, Labbé P, Chandre F, Cohuet A. Malaria vector control still matters despite insecticide resistance. Trends Parasitol. 2017:33:610-8.

6. Alout H, Roche B, Dabiré RK, Cohuet A. Consequences of insecticide resistance on malaria transmission. PLoS Pathog. 2017;13:e1006499.

7. Djogbénou L, Pasteur N, Akogbeto M, Weill M, Chandre F. Insecticide resistance in the Anopheles gambiae complex in Benin: a nationwide survey. Med Vet Entomol. 2011;25:256-67.

8. Dabiré K, Diabaté A, Namountougou M, Toe K, Ouari A, Kengne P, et al. Distribution of pyrethroid and DDT resistance and the L1014F kdr mutation in Anopheles gambiae s.l. from Burkina Faso (West Africa). Trans R Soc Trop Med Hyg. 2009;103:1113-20.

9. Camara S, Koffi AA, Alou LPA, Koffi K, Kabran J-PK, Koné A, et al. Mapping insecticide resistance in Anopheles gambiae (s.l.) from Côte d'Ivoire. Parasit Vectors. 2018;11:19.

10. President's Malaria Initiative (PMI). Twelfth annual report to USA congress. Washington DC: President's Malaria Initiative; 2018.

11. Yadouleton A, Martin T, Padonou G, Chandre F, Asidi A, Djogbenou L, et al. Cotton pest management practices and the selection of pyrethroid resistance in Anopheles gambiae population in northern Benin. Parasit Vectors. 2011:4:60.

12. Diabate A, Baldet T, Chandre F, Akoobeto M, Guiguemde TR, Darriet F, et al. The role of agricultural use of insecticides in resistance to pyrethroids in Anopheles gambiae s.I. in Burkina Faso. Am J Trop Med Hyg. 2002;67:617-22.

13. Abuelmaali SA, Elaagip AH, Basheer MA, Frah EA, Ahmed FT, Elhaj HF, et al. Impacts of agricultural practices on insecticide resistance in the malaria vector Anopheles arabiensis in Khartoum State, Sudan. PLoS ONE. 2013;8:e80549.

14. Nkya TE, Poupardin R, Laporte F, Akhouayri I, Mosha F, Magesa S, et al. Impact of agriculture on the selection of insecticide resistance in the malaria vector Anopheles gambiae: a multigenerational study in controlled conditions. Parasit Vectors. 2014;7:480.

15. WHO. Global plan for insecticide resistance management in malaria vectors. Geneva: World Health Organization; 2012

16. WHO. Framework for a national plan for monitoring and management of insecticide resistance in malaria vectors. Geneva: World Health Organization; 2017.

17. Miles A, Harding N, Bottà $G$, et al. Genetic diversity of the African malaria vector Anopheles gambiae. Nature. 2017;552:96-100.

18. Lucas ER, Rockett KA, Lynd A, Essandoh J, Grisales N, Kemei B, et al. A high throughput multi-locus insecticide resistance marker panel for tracking resistance emergence and spread in Anopheles gambiae. Sci Rep. 2019;9:13335

19. Gillies M, Coetzee M. A supplement to the Anophelinae of Africa south of the Sahara. Publ S Afr Inst Med Res. 1987;55:1-143.

20. Santolamazza F, Mancini E, Simard F, Qi Y, Tu Z, della Torre A. Insertion polymorphisms of SINE200 retrotransposons within speciation islands of Anopheles gambiae molecular forms. Malar J. 2008;7:163.

21. Scott JA, Brogdon WG, Collins FH. Identification of single specimens of the Anopheles gambiae complex by the polymerase chain reaction. Am J Trop Med Hyg. 1993:49:520-9.

22. Assogba BS, Djogbénou LS, Milesi P, Berthomieu A, Perez J, Ayala D, et al. An ace-1 gene duplication resorbs the fitness cost associated with resistance in Anopheles gambiae, the main malaria mosquito. Sci Rep. 2015:5:14529.

23. WHO. Test procedures for insecticide resistance monitoring in malaria vector mosquitoes. Geneva: World Health Organization; 2016

24. Martinez-Torres D, Chandre F, Williamson M, Darriet F, Berge JB, Devonshire AL, et al. Molecular characterization of pyrethroid knockdown resistance $(\mathrm{kdr})$ in the major malaria vector Anopheles gambiae s.s. Insect Mol Biol. 1998;7:179-84.

25. Weill M, Malcolm C, Chandre F, Mogensen K, Berthomieu A, Marquine M, et al. The unique mutation in ace-1 giving high insecticide resistance is easily detectable in mosquito vectors. Insect Mol Biol. 2004;13:1-7.

26. Lebreton J-D, Burnham KP, Clobert J, Anderson DR. Modeling survival and testing biological hypotheses using marked animals: a unified approach with case studies. Ecol Monogr. 1992;62:67-118.

27. Anderson D, Burnham K, White G. AIC model selection in overdispersed capture-recapture data. Ecology. 1994;75:1780-93.

28. Crawley M. The R Book. Chichester: Wiley; 2007.

29. Hochberg Y. A sharper Bonferroni procedure for multiple tests of significance. Biometrika. 1988;75:800-2.

30. Weir BS, Cockerham CC. Estimating F-statistics for the analysis of population structure. Evolution. 1984;38:1358-70.

31. Djègbè $I$, Boussari $O$, Sidick $A$, Martin $T$, Ranson $H_{t}$ Chandre $F$, et al. Dynamics of insecticide resistance in malaria vectors in Benin: first evidence of the presence of L1014S kdr mutation in Anopheles gambiae from West Africa. Malar J. 2011;10:261 
32. Yadouleton AW, Padonou G, Asidi A, Moiroux N, Bio-Banganna S, Corbel $V$, et al. Insecticide resistance status in Anopheles gambiae in southern Benin. Malar J. 2010;9:83.

33. Yadouleton AWM, Asidi A, Djouaka RF, Braïma J, Agossou CD, Akogbeto MC. Development of vegetable farming: a cause of the emergence of insecticide resistance in populations of Anopheles gambiae in urban areas of Benin. Malar J. 2009;8:103.

34. Djènontin A, Bio-Bangana S, Moiroux N, Henry M-C, Bousari O, Chabi J, et al. Culicidae diversity, malaria transmission and insecticide resistance alleles in malaria vectors in Ouidah-Kpomasse-Tori district from Benin (West Africa): a pre-intervention study. Parasit Vectors. 2010;3:83.

35. Aïkpon R, Agossa F, Ossè R, Oussou O, Aïzoun N, Oké-Agbo F, et al. Bendiocarb resistance in Anopheles gambiae s.l. populations from Atacora department in Benin, West Africa: a threat for malaria vector control. Parasit Vectors. 2013;6:192.

36. Yahouédo GA, Cornelie S, Djègbè I, Ahlonsou J, Aboubakar S, Soares $C$, et al. Dynamics of pyrethroid resistance in malaria vectors in southern Benin following a large-scale implementation of vector control interventions. Parasit Vectors. 2016;9:385.

37. Fassinou AJY, Koukpo CZ, Ossè RA, Agossa FR, Assogba BS, Sidick A, et al. Genetic structure of Anopheles gambiae s.s. populations following the use of insecticides on several consecutive years in southern Benin. Trop Med Health. 2019;47:23.

38. Djouaka RF, Bakare AA, Coulibaly ON, Akogbeto MC, Ranson H, Hemingway J, et al. Expression of the cytochrome P450s, CYP6P3 and CYP6M2 are significantly elevated in multiple pyrethroid resistant populations of Anopheles gambiae s.s. from southern Benin and Nigeria. BMC Genomics. 2008;9:538.

39. Sinka ME, Bangs MJ, Manguin S, Coetzee M, Mbogo CM, Hemingway J, et al. The dominant Anopheles vectors of human malaria in Africa, Europe and the Middle East: occurrence data, distribution maps and bionomic précis. Parasit Vectors. 2010;3:117.

40. Moiroux N, Damien GB, Egrot M, Djenontin A, Chandre F, Corbel V, et al. Human exposure to early morning Anopheles funestus biting behavior and personal protection provided by long-lasting insecticidal nets. PLoS ONE. 2014;9:e104967.

41. Dabiré KR, Diabaté A, Namountougou M, Djogbenou L, Wondji C, Chandre $F$, et al. Trends in insecticide resistance in natural populations of malaria vectors in Burkina Faso, West Africa: 10 years'surveys. In: Khan Perveen F, editor. Insecticides. Pest Engineering. London: Intechopen; 2012. p. 479-502.

42. Luc DS, Assogba B, Laurette D, Michel M. Indirect evidence that agricultural pesticides select for insecticide resistance in the malaria vector Anopheles gambiae. J Vector Ecol. 2016;41:34-40.

43. Norris LC, Main BJ, Lee Y, Collier TC, Fofana A, Cornel AJ, et al. Adaptive introgression in an African malaria mosquito coincident with the increased usage of insecticide-treated bed nets. Proc Natl Acad Sci USA. 2015;112:815-20.

44. Alout H, Ndam NT, Sandeu MM, Djegbe I, Chandre F, Dabire RK, et al. Insecticide resistance alleles affect vector competence of Anopheles gambiae s.s. for Plasmodium falciparum field isolates. PLOS ONE. 2013;8:e63849.

45. Ndiath MO, Cailleau A, Diedhiou SM, Gaye A, Boudin C, Richard V, et al. Effects of the kdr resistance mutation on the susceptibility of wild Anopheles gambiae populations to Plasmodium falciparum: a hindrance for vector control. Malar J. 2014;13:340.

46. Kabula B, Tungu P, Rippon EJ, Steen K, Kisinza W, Magesa S, et al. A significant association between deltamethrin resistance, Plasmodium falciparum infection and the Vgsc-1014S resistance mutation in Anopheles gambiae highlights the epidemiological importance of resistance markers. Malar J. 2016;15:289.

47. Churcher TS, Lissenden N, Griffin JT, Worrall E, Ranson H. The impact of pyrethroid resistance on the efficacy and effectiveness of bednets for malaria control in Africa. Elife. 2016;5:e16090.

48. Riveron JM, Huijben S, Tchapga W, Tchouakui M, Wondji MJ, Tchoupo M, et al. Escalation of pyrethroid resistance in the malaria vector Anopheles funestus induces a loss of efficacy of piperonyl butoxide-based insecticide-treated nets in Mozambique. J Infect Dis. 2019:220:467-75.

49. Toe KH, Jones CM, N'Fale S, Ismail HM, Dabire RK, Ranson H. Increased pyrethroid resistance in malaria vectors and decreased bed net effectiveness, Burkina Faso. Emerg Infect Dis. 2014;20:1691-6.
50. Weedall GD, Mugenzi LMJ, Menze BD, Tchouakui M, Ibrahim SS, Amvongo-Adjia N, et al. A cytochrome P450 allele confers pyrethroid resistance on a major African malaria vector, reducing insecticide-treated bednet efficacy. Sci Transl Med. 2019;11:eaat7386.

51. Koffi AA, Alou LPA, Adja MA, Koné M, Chandre F, N'Guessan R. Update on resistance status of Anopheles gambiae s.s. to conventional insecticides at a previous WHOPES field site, "Yaokoffikro", 6 years after the political crisis in Côte d'Ivoire. Parasit Vectors. 2012;5:68.

52. Namountougou M, Simard F, Baldet T, Diabaté A, Ouédraogo JB, Martin T, et al. Multiple insecticide resistance in Anopheles gambiae s.l. populations from Burkina Faso, West Africa. PLoS ONE. 2012;7:e48412.

53. Pocquet N, Darriet F, Zumbo B, Milesi P, Thiria J, Bernard V, et al. Insecticide resistance in disease vectors from Mayotte: an opportunity for integrated vector management. Parasit Vectors. 2014;7:299.

54. Akogbéto MC, Aikpon RY, Azondékon R, Padonou GG, Ossè RA, Agossa $F R$, et al. Six years of experience in entomological surveillance of indoor residual spraying against malaria transmission in Benin: lessons learned, challenges and outlooks. Malar J. 2015;14:242.

55. Assogba BS, Milesi P, Djogbénou LS, Berthomieu A, Makoundou P, BabaMoussa LS, et al. The ace-1 locus is amplified in all resistant Anopheles gambiae mosquitoes: fitness consequences of homogeneous and heterogeneous duplications. PLoS Biol. 2016;14:e2000618.

56. Assogba BS, Alout H, Koffi A, Penetier C, Djogbénou LS, Makoundou P, et al. Adaptive deletion in resistance gene duplications in the malaria vector Anopheles gambiae. Evol. Appl. 2018;1 1:1245-56.

57. Oduola AO, Idowu ET, Oyebola MK, Adeogun AO, Olojede JB, Otubanjo $\mathrm{OA}$, et al. Evidence of carbamate resistance in urban populations of Anopheles gambiae ss mosquitoes resistant to DDT and deltamethrin insecticides in Lagos. South-Western Nigeria. Parasit Vectors. 2012;5:116.

58. Essandoh J, Yawson AE, Weetman D. Acetylcholinesterase (Ace-1) target site mutation 1195 is strongly diagnostic of carbamate and organophosphate resistance in Anopheles gambiae ss and Anopheles coluzzii across southern Ghana. Malar J. 2013;12:404.

59. Alou LPA, Koffi AA, Adja MA, Tia E, Kouassi PK, Koné M, et al. Distribution of ace-1 $R$ and resistance to carbamates and organophosphates in Anopheles gambiae ss populations from Côte d'Ivoire. Malaria J. 2010;9:167.

60. Dabire RK, Namountougou M, Diabaté A, Soma DD, Bado J, Toe HK, et al. Distribution and frequency of kdr mutations within Anopheles gambiae sl populations and first report of the Ace. 1G119S mutation in Anopheles arabiensis from Burkina Faso (West Africa). PLoS One. 2014;9:e101484.

\section{Publisher's Note}

Springer Nature remains neutral with regard to jurisdictional claims in published maps and institutional affiliations.

\footnotetext{
Ready to submit your research? Choose BMC and benefit from:

- fast, convenient online submission

- thorough peer review by experienced researchers in your field

- rapid publication on acceptance

- support for research data, including large and complex data types

- gold Open Access which fosters wider collaboration and increased citations

- maximum visibility for your research: over $100 \mathrm{M}$ website views per year
}

At BMC, research is always in progress.

Learn more biomedcentral.com/submissions 